\title{
Rechtliche Rahmenbedingungen der Anwendung «nicht zugelassener» Arzneimittel ${ }^{1}$
}

Pascal Lachenmeier ${ }^{2}$

1 Eine ausführliche Fassung dieses Textes mit weiteren Hinweisen ist am 11. Mai 2009 in Jusletter erschienen.

2 Der Autor war wissenschaftlicher Assistent an der Juristischen Fakultät de Universität Basel und dissertierte zu einem medizinrechtlichen Thema.

3 Wegen besserer Lesbarkeit wird die männliche Form verwendet, Frauen sind ebenfalls gemeint.

Korrespondenz:

Dr. iur. Pascal Lachenmeier

Muttenzerstrasse 36

CH-4127 Birsfelden
Man sollte meinen, dass der Einsatz eines Heilung versprechenden Arzneimittels bei einer lebensbedrohenden Erkrankung nicht beschränkt sein kann, solange die Sicherheit des Arzneimittels in einer befriedigenden Art und Weise gewährleistet ist. Schliesslich geht es für die betroffene Person um Leben und Tod. Dennoch bewegen wir uns in einem hochkomplexen Bereich des Medizinrechts. Den dieses Gebiet durchziehenden Prämissen soll hier nachgegangen werden.

\section{Medizin- und arztrechtliche Rahmen- bedingungen}

\section{Behandlungs- und Selbstbestimmungsrecht des Patienten ${ }^{3}$}

Im Zentrum steht der Patient mit seiner schweren, lebensbedrohenden Erkrankung, dessen Binnenperspektive auf die Problematik eine fundamental andere sein kann als diejenige der übrigen Bevölkerung. Die Behandlung mit einem Arzneimittel in der Onkologie kann den Patienten vor eine Alles-oder-Nichts-Situation stellen. Der Behandlungsanspruch des lebensbedrohten Patienten muss im Lichte des verfassungsrechtlich garantierten Lebensrechtes (Art. 10 Abs. 1 BV) und des Schutzes seiner Gesundheit (als Teilgehalt der persönlichen Freiheit, Art. 10 Abs. 2 BV) gesehen werden. Der hohe Rang dieser Rechtsgüter bedingt den Anspruch des Einzelnen, dass staatliche Regelungen, die diesen Behandlungsanspruch begrenzen, besondere Begründungsqualität aufweisen. Ein umfassendes Grundrecht auf Gesundheit ist jedoch umstritten. Ein positives Leistungsrecht im Sinne eines sozialen Grundrechtes existiert nicht. Immerhin scheint die freie Wahl eines Arzneimittels, als Ausfluss aus dem tendenziell sich entwickelnden Recht auf Gesundheit, garantiert zu sein.

\section{Informed consent}

Mit dieser Problematik hängt der informed consent eng zusammen. Die informierte Zustimmung dient sowohl dem Interesse des Patienten auf Information als auch dem Interesse des behandelnden Arztes auf Schutz vor strafrechtlicher und zivilrechtlicher Haftung. Im hier diskutierten Bereich der Anwendung von Arzneimitteln ausserhalb des ordentlichen Zulassungsrahmens kommt der informierten Zustimmung eine zentrale Bedeutung zu. Ausgehend vom Grund-
Off-label use - exceptions prévues par la loi sur les produits thérapeutiques (LPTh) Le diagnostic d'une maladie menaçant la vie du patient le confronte à la question de la disponibilité des médicaments. À cette question est liée le contrôle des procédures d'admission des médicaments et de leurs exceptions prévues par la loi sur les produits thérapeutiques (LPTh) et par la pratique de Swissmedic au regard des principes généraux du droit sur les médicaments. Mis à part le «off-label use» relativement connu, de nombreuses exceptions plus complexes existent. Leurs fondements légaux et pratiques doivent être remis en question en vue des prémisses reconnues du droit médical comme par exemple le droit à l'autodétermination du patient ou la liberté thérapeutique du médecin.

satz der Verfügungsfähigkeit des Einzelnen über seine körperliche Integrität, die ihre Begrenzung in einer (umstrittenen) Verfügungsunfähigkeit über sein Leben findet, bleibt es dem Patienten freigestellt, eine Therapie zu wählen, solange diese nicht schädlich (primum non nocere) oder gar tödlich ist. Hier ist festzuhalten, dass die Grenzen zwischen Schaden und Heilen bei Therapien in der Onkologie in dem Masse zunehmend verwischen, als die Krankheit fortgeschritten ist und mindestens als ultima ratio, als letztes Mittel, eine Therapie zulässig erscheinen kann, die bei nicht lebensbedrohenden Krankheiten eher einem Schaden entsprechen würde. In der onkologischen Behandlung, und bei einem Heilversuch generell, kann bildlich von einer Art Schicksalsgemeinschaft zwischen Arzt und Patient gesprochen werden. Trotzdem ist der Patient so zu informieren, dass er die ihm zustehende Entscheidungsmacht ausüben kann.

\section{Therapiefreiheit und Sorgfaltspflicht des Arztes} Der Arzt verfügt über eine verfassungsrechtlich geschützte Therapiefreiheit. Die Therapiefreiheit des Arztes stellt wie das Selbstbestimmungsrecht des Patienten eine grundsätzliche Hürde für die Einschränkung der Freiheit der Therapiewahl dar. Der Arzt ist sowohl berechtigt als auch verpflichtet, unter den zur Verfügung stehenden Therapien die beste zur Anwendung zu bringen. 


\section{Öffentliches Interesse}

\section{an einem sicheren Arzneimittelmarkt}

Begrenzend wirkt das öffentliche Interesse an einem sicheren Arzneimittelmarkt. Das Interesse der Mehrheit, keine potentiell unsicheren oder unwirksamen Medikamente verabreicht zu bekommen, kann mit dem Interesse des Einzelnen, an einer lebensbedrohlichen Krankheit Leidenden kollidieren. Die unterschiedlichen Situationen erfordern ein abwägendes System. Die Sicherheitsbedürfnisse der Gesellschaft stehen dort zur Disposition, wo der autonome Patient, begleitet durch das Fachwissen seines Arztes, sich dafür entscheiden sollte, ein vertretbares Risiko einzugehen, um einer lebensbedrohlichen Erkrankung zu begegnen. Paternalistische und, wenn gewisse Grenzen nicht eingehalten werden, auch wirtschaftliche Gründe müssen zurückgestellt werden.

\section{Finanzielle Aspekte und Arzneimittelmarkt}

Die Frage der Finanzierbarkeit der Therapie kann die Frage ihrer Verfügbarkeit an Bedeutung überlagern. Trotzdem sind die Fragenkomplexe vorerst zu trennen. Wirtschaftliche Überlegungen haben auch einen Einfluss auf die Verfügbarkeit von Medikamenten. Je breiter die angemeldete Indikation ausfällt, desto höher sind die Kosten für die Zulassung, resp. die klinische Testphase des Arzneimittels.

\section{Zulassungserfordernis als Grundsatz des Heilmittelgesetzes (HMG)}

Das HMG stellt in erster Linie ein Polizeigesetz dar. Die Versorgung der Bevölkerung mit qualitativ hochstehenden, sicheren und wirksamen Heilmitteln steht im Vordergrund. Das Schweizerische Heilmittelinstitut (Swissmedic) ist zentrale Zulassungsinstanz. Es erteilt nicht nur Zulassungen gemäss Art. 9 HMG und Sonderbewilligungen gemäss Art. 9 Abs. 4 HMG, sondern ist auch Urheber der Pharmakopöe, deren Anforderungen die Arzneimittel ebenfalls zu entsprechen haben. Die Spezialitätenliste stellt die Schnittstelle zwischen dem HMG und dem Krankenversicherungsgesetz (KVG) her. Gemäss Art. 25, 32 und 52 KVG umfasst die Pflichtleistung in der Grundversicherung grundsätzlich nur die auf der Spezialitätenliste aufgeführten (zugelassenen) Arzneimittel. Mit der Aufnahme eines Arzneimittels in die Spezialitätenliste wird derselben die Tauglichkeit unter den Aspekten der Wirksamkeit, Zweckmässigkeit und Wirtschaftlichkeit attestiert.

\section{Ausnahmen von der ordentlichen Zulassung}

Einige Ausnahmen vom Grundsatz des Zulassungserfordernisses definiert das HMG in Art. 9 Abs. 2 selbst. Die nicht definierten Formen (z. B. off-label use) lässt das HMG unter Berücksichtigung der Sorgfaltspflichten von Art. 3 und 26 HMG grundsätzlich zu. Die Unterscheidung der einzelnen Gruppen von «nicht zugelassenen» Arzneimitteln ist nicht ganz einfach, weshalb sie vorgängig definiert werden sollen ${ }^{4}$.

\section{Off-label use}

Mit Abstand am häufigsten, gerade auch im pädiatrischen Bereich, tritt der «Off-label use» auf. Darunter versteht man die Anwendung gebrauchsfertiger Arzneimittel ausserhalb ihrer zugelassenen Indikation.

\section{Unlicensed use}

Die Begriffe des unlicensed use wie auch des folgenden compassionate use werden häufig nicht trennscharf unterschieden. Unter unlicensed use ist die Anwendung eines Arzneimittels zu verstehen, das zwar in gebrauchsfertigem Zustand vorliegt, aber keine schweizerische Zulassung aufweist. Denkbar ist zudem auch ein off-label use eines unlicensed use. Dann wird ein im Ausland zugelassenes Arzneimittel ausserhalb der dort zugelassenen Indikation angewendet (im Sinne eines sog. Last-hope-Medikaments). Art. 36 AMBV 5 verlangt als Voraussetzung für den unlicensed use eine Sonderbewilligung von Swissmedic.

Die Voraussetzungen gemäss Praxis der Swissmedic sind folgende:

- Fehlende Alternative in der Schweiz (kein zugelassenes Präparat mit gleicher Indikation, schlechteres Nutzen-Risiko-Verhältnis eines zugelassenen Präparates, Alternativtherapie wurde ohne Erfolg durchgeführt)

- Keine Rücknahme vom Markt wegen Sicherheitsbedenken

- Zulassung durch anerkannte Zulassungsbehörde im Drittland, jedoch kein off-label use

- Einsatz bei einem einzelnen, namentlich bekannten Patienten

- Die Bewilligung wird an einen einzelnen, verantwortlichen Arzt erteilt

- Meldepflicht gemäss Art. 59 HMG besteht (Meldung unerwünschter Wirkungen und Vorkommnisse)

- Die Bewilligung wird für eine begrenzte Arzneimittelmenge und zu einer begrenzten zeitlichen Anwendung erteilt

- Der Patient muss informiert werden und eine schriftliche Einwilligungserklärung abgeben

- Der Versicherungsschutz ist über die Privathaftpflichtversicherung des Arztes gewährleistet.

\section{Compassionate use}

Unter compassionate use wird ein spezieller Fall der Anwendung eines noch nicht zugelassenen Arzneimittels verstanden. Aus «Mitleid» wird zwingend befristet die Anwendung eines solchen noch nicht zugelassenen Arzneimittels bewilligt (Sonderbewilligung). Art. 9 Abs. 4 HMG stellt folgende Bedingungen auf:

- Lebensbedrohende Krankheit

- Vereinbarkeit mit dem Schutz der Gesundheit

- Grosser therapeutischer Nutzen

- Kein vergleichbares Arzneimittel steht zur Verfügung. 
Art. $18 \mathrm{VAZV}^{6}$ konkretisiert die Voraussetzungen von

Art. 9 Abs. 4 HMG:

- Vorliegen muss die Behandlung einer Krankheit, die bei einem spontanen Verlauf in der Regel eine schwere chronische Invalidität zur Folge hat oder kurzfristig zum Tod eines Patienten führt

- Für die Krankheit ist kein gleichwertiges Arzneimittel in der Schweiz zugelassen (resp. nicht verfügbar)

- Die Verfahren der ordentlichen Zulassung würden so lange dauern, dass dadurch irreversible Schädigungen auftreten oder sich verstärken würden.

Die Zulassung für den compassionate use weist dieselben Rechtswirkungen wie die ordentliche Zulassung auf. Sie ermöglicht damit auch einen off-label use der mit der Sonderbewilligung zugelassenen Arzneimittel. Die Voraussetzungen, die gemäss Praxis der Swissmedic erfüllt sein müssen, sind folgende:

- Der Patient leidet an schwerer, potentiell lebensbedrohender oder invalidisierender Krankheit

- Fehlende Alternative in der Schweiz (kein zugelassenes Präparat mit gleicher Indikation, schlechteres Nutzen/Risiko-Verhältnis eines zugelassenen Präparates, Alternativtherapie wurde ohne Erfolg durchgeführt)

- Primär handelt es sich um Notfallinterventionen, um eine letzte Therapiemöglichkeit (last hope)

- Die Arznei wurde in einem Drittland zugelassen oder befindet sich im Zulassungsverfahren. Ist weder das eine noch das andere der Fall, sind solide wissenschaftliche Resultate aus klinischen Versuchen sowie gute Produkteinformationen des Herstellers unabdingbar

- Einsatz bei einem einzelnen, namentlich bekannten Patienten

- Die Bewilligung wird an einen einzelnen, verantwortlichen Arzt erteilt

- Ws besteht Meldepflicht gemäss Art. 59 HMG (Meldung unerwünschter Wirkungen und Vorkommnisse)

- Patient muss informiert werden und eine schriftliche Einwilligungserklärung abgeben

- Der Versicherungsschutz ist über die Privathaftpflichtversicherung des Arztes gewährleistet.

\section{Rechtliche Probleme}

\section{Beurteilung des off-label use}

\section{Zulässigkeit der Anwendung off-label}

Der off-label use von Arzneimitteln ist unter den Voraussetzungen von Art. 3 und 26 HMG grundsätzlich zulässig. Dabei handelt es sich um die Berücksichtigung der Sorgfaltspflicht und der anerkannten Regeln der medizinischen und pharmazeutischen Wissenschaften. Die Verwendung off-label kann medizinisch indiziert sein, nämlich dann, wenn sie zur good medical practice, zur Behandlung lege artis gehört.

\section{Bestrebungen nach Klassifizierung}

Vereinzelt wird eine Liste für die Anwendung offlabel gefordert. So verständlich dieses Anliegen erscheint, es besteht Grund zur Zurückhaltung. Wenn neben der ordentlichen Zulassung, den vielfältigen Möglichkeiten der vereinfachten Zulassung und der eigentlichen Spezialitätenliste auch noch eine Spezialitätenliste off-label geführt wird, drohen parallele Standards.

\section{Finanzierung und Spezialitätenliste}

Hierbei handelt es sich um eine Frage des KVG. Die Vergütung der Kosten für einen off-label use ist grundsätzlich nicht durch die Krankenkassen zu übernehmen. Ausnahmen sind gemäss Praxis des Bundesgerichts das Arzneimittel als unerlässlicher Bestandteil eines von der Krankenkasse zu erstattenden Behandlungskomplexes oder das off-label angewendete, grundsätzlich auf der Spezialitätenliste figurierende Arzneimittel, das gegen eine lebensbedrohliche Krankheit eingesetzt wird, dessen Einsatz mit dem Schutz der Gesundheit vereinbar ist, von dem ein grosser therapeutischer Nutzen zu erwarten ist, wobei kein vergleichbares Arzneimittel zur Verfügung steht.

\section{Beurteilung des unlicensed use}

\section{Grundsätzliches}

Unlicensed use eines Arzneimittels bedeutet, dass ein zulassungsbedürftiges Arzneimittel, das in der Schweiz keine Zulassung aufweist, trotzdem angewendet wird. Dabei handelt es sich nicht um unsichere, ungeprüfte Medikamente, wie der Begriff suggerieren könnte, sondern die Swissmedic bewilligt im Sonderbewilligungsverfahren die Anwendung von im Ausland zugelassenen Medikamenten. Das Zulassungsregime des HMG und der Verordnung des Bundesrates (AMBV) fällt sehr strikt aus. So wird zwar ein Medikament innerhalb der Indikation einer anerkannten ausländischen Zulassungsbehörde zugelassen, nicht jedoch ein offlabel use.

\section{Sonderbewilligungspraxis der Swissmedic}

\section{Fehlende Alternative}

Im Lichte der vorgestellten Aspekte erscheint es problematisch, vom Patienten das Ausweichen auf eine alternative Behandlungsmethode $\mathrm{zu}$ verlangen. Insbesondere ist nicht klar, was als Alternative gelten soll, ob z. B. ein anders wirkendes Medikament überhaupt eine Alternative darstellt.

\section{Off-label use des unlicensed use}

Die Zumutbarkeit des Verbots des off-label use eines unlicensed use steht in Frage. Hier hätte es genügt, dem Gesuchssteller den Nachweis der wissenschaftlichen Begründbarkeit des off-label use im Ausland aufzuerlegen. rischen über die vereinfachte Zulassung von Arzneimitteln und die Zulassung von (VAZV) 
Begrenzung auf Zeit und Menge

Um der Therapiefreiheit des Arztes genügend Raum zu lassen, kann diese Begrenzung in zeitlicher und mengenmässiger Hinsicht lediglich einen Rahmen abstecken, innerhalb dessen sich der Arzt selbst um die konkrete zeitliche Ausdehnung der Anwendung des Arzneimittels bemüht.

\section{Grundsatz der Selbstfinanzierung}

Nach der Rechtsprechung des Bundesgerichts ist davon auszugehen, dass ein unlicensed use durch den Patienten selber finanziert werden muss. Diese Einschränkung wirkt als faktische Begrenzung der Verfügbarkeit von Arzneimitteln in der unlizenzierten Anwendung stark auf den Sonderbewilligungskatalog der Swissmedic zurück. Damit wird ersichtlich, wie therapieverhindernd diese Praxis ausgestaltet ist und wie zurückhaltend die Interpretation der einzelnen limitierenden Voraussetzungen vorgenommen werden sollte, soll nicht ein verbotsähnlicher Tatbestand geschaffen werden.

\section{Beurteilung des compassionate use}

\section{Mitleid, Notstand, letzte Chance?}

Die Rede vom «compassionate use» ist grundsätzlich kritisch zu hinterfragen. Einerseits steht in Frage, welche Konsequenzen (dieses) «Mitleid» haben soll. Andererseits ist der hinter einem solchen Mitleidsbekunden stehenden Haltung mit Skepsis zu begegnen, weil sie einem eher veralteten ärztlich-christlich-paternalistischen Schema entspricht.

\section{Voraussetzungen des compassionate use}

Im Unterschied zum unlicensed use muss beim compassionate use eine schwere, potentiell lebensbedrohende oder invalidisierende Krankheit vorhanden sein, eine Zulassung im Ausland ist nicht erforderlich und der off-label use ist grundsätzlich zulässig. Gemäss bundesgerichtlicher Rechtsprechung, soll ein compassionate use grundsätzlich zurückhaltend bewilligt werden, weil ansonsten der Zweck der generellen Zulassungspflicht unterwandert werden könnte. Allerdings ist dem HMG eine Grundlage für eine restriktive Praxis nicht zu entnehmen. Bei Vorliegen der Voraussetzungen ist also eine Bewilligung zu erteilen. Die Zulassung per Sonderbewilligung wird an sehr enge Voraussetzungen geknüpft.

\section{Diskussion einzelner Voraussetzungen}

\section{Lebensbedrohende/invalidisierende Krankheit}

Dass es sich beim compassionate use um Krankheiten handeln muss, die potentiell lebensbedrohend sind, kann gut begründet werden. Im Extremfall weist das Arzneimittel weder in der Schweiz noch im Ausland die erforderliche Zulassung auf. Daher ist eine Sonderbewilligung, in Anbetracht der Gefahren, die mit der Behandlung auftreten können, mit der Einschränkung auf lebensbedrohende bzw. invalidisierende
Krankheiten zulässig. Schwieriger zu begründen sind Voraussetzungen wie jene, dass andere Behandlungen bereits fehlgeschlagen sind und nunmehr die letzte therapeutische Rettungsmöglichkeit ansteht (last-hope). Es ist aber fraglich, ob es dem Erkrankten zumutbar ist, andere Therapien zu absolvieren, wenn er eine Therapie im Sinne des compassionate use auch sofort in Angriff nehmen kann.

\section{Fehlende Alternative, grosser Nutzen}

In diesem Punkt widersprechen die Erläuterungen der Swissmedic dem HMG. Die Vorgaben des HMG in Art. 9 Abs. 4 werden unterlaufen. Denn im Gesetz wird ein grosser therapeutischer Nutzen verlangt, während die Swissmedic in ihren Erläuterungen, gleich wie beim unlicensed use, lediglich verlangt, dass es an einer den gleichen Erfolg versprechenden Alternative fehlt. Das Erfordernis des grossen therapeutischen Nutzens dürfte über dasjenige des Fehlens einer gleichwertigen Alternative weit hinausgehen. Gleichwohl wäre dem Wortlaut der Erläuterung gegenüber dem Gesetzeswortlaut der Vorzug zu geben, weil sie dem Behandlungsrecht des Patienten und der Therapiefreiheit des Arztes besser entspräche. Der potentiell in seinem Leben Bedrohte muss nicht darlegen, dass die Alternative im Sinne des compassionate use einen grossen therapeutischen Nutzen erbringt, sondern lediglich, dass er bei inländisch erhältlichen Alternativen auf einen (unzumutbaren) schlechteren Weg verwiesen würde.

\section{Zulassung, Möglichkeit zusätzlicher Auflagen}

Die Kombination einer ausländischen Zulassung (durch eine anerkannte Zulassungsbehörde) mit dem zuvor erwähnten grossen therapeutischen Nutzen, der resultieren muss, stellt eine zu hohe Hürde dar. Ebensowenig nachvollziehbar ist die Möglichkeit zusätzlicher Auflagen, weil diese zur Rechtsunsicherheit beiträgt. ${ }^{7}$

\section{Schluss}

Es erscheint fraglich, ob die geltenden Rechtsgrundlagen eine adäquate Lösung bereithalten. Insbesondere die einschränkenden Vorschriften der Art. 9 Abs. 4 HMG i.V.m. Art. 18 f. VAZV sollten, im Lichte der Autonomie des Patienten und der Therapiefreiheit des Arztes betrachtet, nochmals auf den Prüfstand. Weshalb nach diesen Vorschriften der aufgrund eines rechtskonformen informed consent in die Behandlung einwilligende Patient auf einen schlechteren Weg verwiesen wird, leuchtet nicht ein. Etwas anderes kann grundsätzlich für die Frage der Vergütung der Therapie durch die Krankenkasse gelten. Das Solidaritätsprinzip übt hier einen starken Einfluss aus. Diese Einschränkung ist aber nicht so einschneidend wie jene, dass ein Arzneimittel gar nicht angewendet werden darf. Die Hürden für letztere Einschränkung sind deshalb sehr genau auf ihre Kompatibilität mit den rechtsmedizinischen Grundsätzen zu überprüfen. 